IJBPAS, April, 2019, 8(4): 800-814

ISSN: $2277-4998$

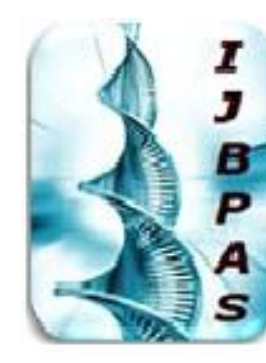

International Journal of Biology, Pharmacy and Allied Sciences (IJBPAS) 'A B ridge Between Caboratory and Qundo'

Www.iibpas.com

DOWN-REGULATION OF ANTIOXIDATIVE VARIABLES AND THEIR INTERACTIVE ROLE IN THE DISEASE PROGRESSION IN LEUKEMIC PATIENTS

\title{
SHAFAQ $M^{2}$, IMRAN $M^{3}, \operatorname{ASLAM~} \mathbf{N}^{4}$, WAQUAR $\mathbf{S}^{1}, \operatorname{HUSSAIN~}^{1}, \operatorname{ALI} \mathbf{Q}^{1}$ AND MALIK A 1* $^{\text {* }}$
}

1: Institute of Molecular Biology and Biotechnology (IMBB), The University of Lahore-Pakistan

2: Bakhtawar Amin Medical and Dental College, Multan-Pakistan

3: Nishtar Medical College, Multan-Pakistan

4: Independent Medical College, Faisalabad-Pakistan

*Corresponding Author: Arif Malik (Ph.D): E Mail: arifuaf@yahoo.com; Cell: 0321-

8448196; Tel: +92 42-7515460-7, Fax: +92-42-7515519

Received 25 ${ }^{\text {th }}$ Nov. 2018; Revised $16^{\text {th }}$ Dec. 2018; Accepted $29^{\text {th }}$ Dec. 2018; Available online $1^{\text {st }}$ April 2019

https://doi.org/10.31032/IJBPAS/2019/8.4.4695

\section{ABSTRACT}

BACKGROUND: Leukemia is most prevalent in children and is the second most common reason of mortality in children aged between 1-14 years. It accounts for every 3 of the 10 cancers diagnosed in children aged below 15 years. Exact causes of leukemia are still unknown. Evidence suggests certain cellular, genetic factors and biochemical alterations are responsible for the disease

MATERIAL AND METHOD: Total 70 individuals were taken in present study in which 50 included in positive control and other 20 were included in negative control group. The $5 \mathrm{ml}$ blood sample was collected from each individual to perform following biomarkers through specific respective protocols. Antioxidants and MDA assessed through specterophometery 
method but the levels of S ferritin, S Fe, IL-6, hs-CRP and TNF- $\alpha$ were determined through commercially available ELIZA kits.

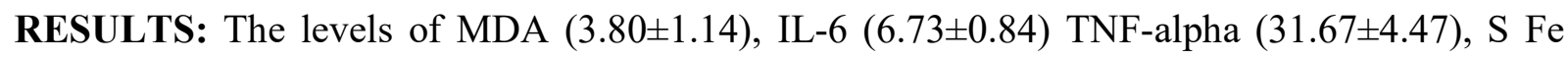
(138.16 \pm 19.06$)$ and $S$ ferritin $(186.29 \pm 69.14)$ were increased in subjects as compared to normal persons $(1.43 \pm 0.36),(5.64 \pm 0.51),(29.90 \pm 1.142),(85.28 \pm 3.26)$ and $(105.57 \pm 1.95)$ respectively. The levels of antioxidant like SOD (0.09 \pm 0.091$)$, CAT (2.25 \pm 1.18$)$, GSH (4.35 \pm 1.62$)$, GSH-Px $(6.61 \pm 0.37)$ were decrease in leukemic patients but the levels of GSH-Pr $(4.39 \pm 1.59)$ increased in same individuals as compared to normal control group $(0.49 \pm 0.14),(3.95 \pm 0.81),(9.79 \pm 1.22)$, $(8.22 \pm 0.69)$, and $(1.47 \pm 0.22)$ respectively. Levels of GGT (58.75 \pm 8.56$)$ and hs-CRP $(1.48 \pm 0.301)$ were increased in leukemic patients as compared to control group (43.12 \pm 6.82$)$, $(1.043 \pm 0.023)$.

CONCLUSION: Leukemia are neoplastic disorder, there are several cellular and genetic reasons involved in the progression of the disease. Out of which NK cells reactivity and defective mode of action may contribute as the reason for the leukemia in number of patients. Our study concludes and signifies the importance of antioxidant profile in the progression of disease. As in the subjects having, the disorder showed significant decrease in their antioxidant levels such SOD, GSH, CAT and had increased lipid peroxidation, and increased levels of MDA and lipid profile.

Keywords: Leukemia, Oxidative stress, Antioxidants, Neoplastic Disorders

\section{INTRODUCTION}

Leukemia is a hematological malignancy i.e. that starts form the blood progenitor cells (bone marrow or immune cells). It is divided into different sub-classes i.e. acute, chronic on the base of severity of disease, lymphoblastic, and myelogenous on base of origin and source of progression of the disease [1]. Acute leukemia is a progressive disorder that featured by approx. $20 \%$ myeloblasts in blood or in the bone marrow. Hematological malignancies are such cancers that initiate in the blood progenitor cells i.e. cells of bone marrow or immune system. Myeloproliferative neoplasms (MPNs) are hematologic cancers that ascend from progenitor cells of myeloid hematopoietic malignancy which results in excessive and uncontrolled growth of one type or combination of different types of cells e.g. RBCs, WBCs or platelets. This massive production of cells can be abnormal or immature reading to further clinical 
complications [2]. Leukemia is most and specialized mature blood cells. prevalent in children and it is the second Hematopoietic stem cells are highly most common reason of mortality in children aged between 1-14 years. It accounts for every 3 of the 10 cancers diagnosed in children aged below 15 years [3]. Exact cause of leukemia is still unknown. Evidence suggests certain cellular, genetic factors and biochemical alterations are responsible for the disease [4].

Hematopoiesis is the process of generating all lineages of blood cells by a stepwise manner. These blood cells are synthesized from immature cells in the bone marrow and then discharge into the blood circulatory system as well as in the peripheral organs for further effector functions and maturation steps. In this process the hematopoietic stem cells (HSCs) are the selfrenewing cells which are capable for the production of all lineages of blood cells. Basically hematopoietic stem cells (HSCs) are exhibit in endosteal regions of bone marrow which consist of multiple types of cells that influence directly and indirectly the localization, differentiation and self-renewal of hematopoietic stem cell (HSC). These cells give rise to multipotent progenitors (MPPs) that are non self-renewal cells and further differentiate into more lineage progenitors which are highly differentiated regulated by modulation of apoptosis, proliferation and retention in bone marrow microenvironment. Usually the cancer in the blood occurs due to the result of pathogenic events which interrupt the physiologically homeostatic balance in the mature hematopoietic stem cells of bone marrow. The hematological malignancies are the heterogeneous group of disorders in which abnormal hematopoietic processes are initiated through leukemic stem cells (LSCs). Recent studies suggest that Natural Killer (NK) cell reactivity or its defective mode of action at the receptor level, cognate ligand defectiveness involved to cause the leukemia [5]. Accumulation of differentiation factors i.e. blast and uncontrolled proliferation leads to hematopoietic malignancy which is the most important characteristic of AML (acute myeloblastic leukemia) [6]. Acute lymphocytic leukemia is $5 \mathrm{X}$ more recurrent than AML and accounts for $78 \%$ of all children leukemias diagnosed [7]. ALL has proved itself an incurable disease because of its well developed resistance towards the treatment. Resistance to chemotherapy can be a result of several factors i.e. oxidative stress, formation of ROS, presence of hypodiploid cells. The prevalence of acute 
lymphocytic leukemia in elder patients in every 100,000 patients is 1.0 to 1.6 , which is higher as compared to patients, aged 25-54 (0.6 to 0.7$)$ as reported by surveillance epidemiology and end result study [8].

Mitochondria are specific organelles which play important role in cellular respiration and apoptosis. In a number of researches it is reported that mitochondrial DNA alteration is present in oncogenesis. Research indicates that in leukemic patients mitochondrial DNA alteration, mitochondrial transcription factor $\mathrm{A}$ and increased oxidative stress could be used as molecular signature of leukemia [9]. They play role in signaling process either intracellular or extracellular, exogenously or endogenously. Endogenously they are produced by different processes inside the human body as a by-product of the metabolism, electron transport chain, pollutants and radiation and chemical compounds commonly called as xenobiotics. Antioxidants provide a basic shield against oxidative stress [10]. Oxidative stress which is caused by ROS is responsible for DNA damage, because in normal conditions DNArepair mechanism is functional to repair the DNA. DNA damage is not repaired properly during oxidative stress condition that leads to cause mutagenesis [11]. Biochemical reactions that are initiated by free radicals alter cell permeability, denature proteins, alter enzyme activity, decrease neurotransmitter transmission, cause breakage of DNA and degrade structural proteins. They are also responsible to cause defective signaling pathways, which are normally responsible for the growth and proliferation and differentiation in normal conditions [12]. Antioxidants are important to regulate the oxidative stress which is the important defense mechanism in normal conditions e.g. $\mathrm{Zn}$ and selenium ions are involved in protection against oxidative stress and OS controls formation of free radicals which is a leading cause of pathogenesis in a number of diseases including leukemia [13]. Catalase, MDA (Malondialdehyde) and other stress markers have been observed in a number of blood samples extracted from various diseased patients which are affected by either AML, ALL, HD and lymph cancers and cancers of visceral organs [14]. With the help of pharmacological Ascorbate and redox active quionone which are directly involved to kill the cancer cells separating from normal cells by necrosis. In active and initial phase of acute leukemia levels of glutathione are significantly higher than the retardation phase. Hence levels of glutathione can be used to monitor relapse of disease [15]. 


\section{MATERIALS AND METHODS}

\section{PLACE OF WORK}

The whole experimental work was done in the institute of Molecular Biology and Biotechnology, The University of Lahore, Lahore Pakistan.

\section{EXPERIMENTAL DESIGN}

To find out the role of circulating biochemical markers which were of medical importance and had anti-oxidative capacity in leukemia patients. Total 70 individuals were taken in present study in which 50 included in positive control and other 20 were included in negative control group. The $5 \mathrm{ml}$ blood sample was collected from each individual to perform following biomarkers through specific respective protocols.

\section{BLOOD ANALYSIS}

Blood was centrifuged at $4000 \mathrm{rpm}$ for 10 minutes and serum was separated. Blood samples were collected into EDTA tubes from fasting proteins.

\section{BIOCHEMICAL ANALYSIS}

ESTIMATION OF ANTIOXIDANTS THROUGH VARIOUS PROTOCOLS

SOD was measured by spectrophotometric method of Kakkar et al method. CAT was measured by spectrophotometric method of Aebi method. Glutathione peroxidase activity was measured by methods of Aydin et al., [16]
ESTIMATION

OF

MALANODIALDEHYDE (MDA)

MDA was measured by spectrophotometric method of Okawa et al., method [17].

\section{ESTIMATION OF GAMMA \\ GLUTAMYL TRANFERASE $(\gamma$-GT) IN SERUM PRINCIPLE}

Gamma glutamyl tranferase in serum and liver was estimated by the method of Persijin and van der Slik [18].

\section{ESTIMATION OF GLUTATHIONE REDUCTASE (GR)}

Glutathione reductase was evaluated by using method of David and Richard [19].

\section{DETERMINATION OF FERRITIN}

Serum ferritin was measured by using assay kit Products of Fuji Rebio Incorporated (Tokyo, Japan) and Denka Seiken, Tokyo, Japan [20].

\section{DETERMINATION OF C- REACTIVE PROTEIN (CRP)}

C-Reactive protein (CRP) was determining by CRP Latex test kit [21].

\section{DETERMINATION OF IL-6}

The interleukin- 6 concentration was assessed by the quantitative ELISA (Enzyme Linked Immunosorbent Assay) as mentioned by their manufacturers' protocols (R\&D Systems, Minneapolis, MN, USA) [22]. 


\section{DETERMINATION OF TNF- $\alpha$}

The levels of tumor necrosis alpha (TNF- $\alpha$ ) were determined using commercially available ELISA kits (Affimatrix, Japan) and was expressed in units (pg/ml).

\section{RESULTS}

As shown in present results (Figure 1 A-T) the levels of oxidative biomarkers significantly increased in the leukemic patients as compare to normal healthy individuals because the rate of damage were increased within the time. The levels of MDA (3.80 \pm 1.14$),$ IL-6 (6.73 \pm 0.84$)$ TNFalpha (31.67 \pm 4.47$), \mathrm{S} \mathrm{Fe}(138.16 \pm 19.06)$ and $S$ ferritin $(186.29 \pm 69.14)$ were increased in subjects as compare to normal persons (1.43 \pm 0.36$), \quad(5.64 \pm 0.51), \quad(29.90 \pm 1.142)$, $(85.28 \pm 3.26)$ and $(105.57 \pm 1.95)$ respectively. The $\mathrm{P}$ value also highly significant $\mathrm{P} \leq 0.05$. according to the results of current study the levels of antioxidants also decrease in leukemic patients due to this the rate of

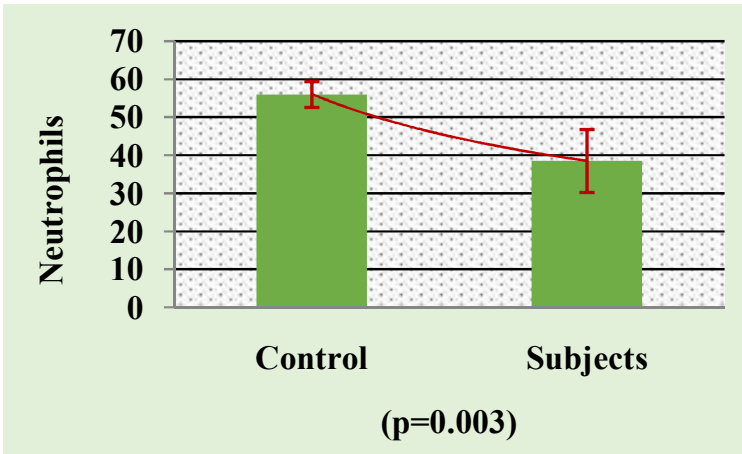

$\mathbf{A}$

IJBPAS, April, 2019, 8(4) oxidative damage were increased because antioxidants have the great potential to neutralize the oxidative damage effect through free radicals production in OS condition. So, the levels of antioxidant like SOD (0.09 \pm 0.091$),$ CAT $(2.25 \pm 1.18)$, GSH (4.35 \pm 1.62$), \quad$ GSH-Px $\quad(6.61 \pm 0.37) \quad$ were decreased in leukemic patients but the levels of GSH-Pr (4.39 \pm 1.59$)$ increased in same individuals as compared to normal control group $(0.49 \pm 0.14),(3.95 \pm 0.81),(9.79 \pm 1.22)$, $(8.22 \pm 0.69)$, and $(1.47 \pm 0.22)$ respectively. The $\mathrm{P}$ values were also highly significant for all of these variables at $\mathrm{P} \leq 0.05$. Like inflammatory biomarkers the levels of GGT (58.75 \pm 8.56$)$ and hs-CRP $(1.48 \pm 0.301)$ were increased in leukemic patients but $\mathrm{Hb}$ $(10.69 \pm 1.87)$ levels were also dropped in same group members as compared to control group (43.12 \pm 6.82$), \quad(1.043 \pm 0.023)$ and $(14.11 \pm 0.87)$ respectively. The $\mathrm{P}$ values of this variables also highly significant at $\mathrm{P} \leq 0.05$.

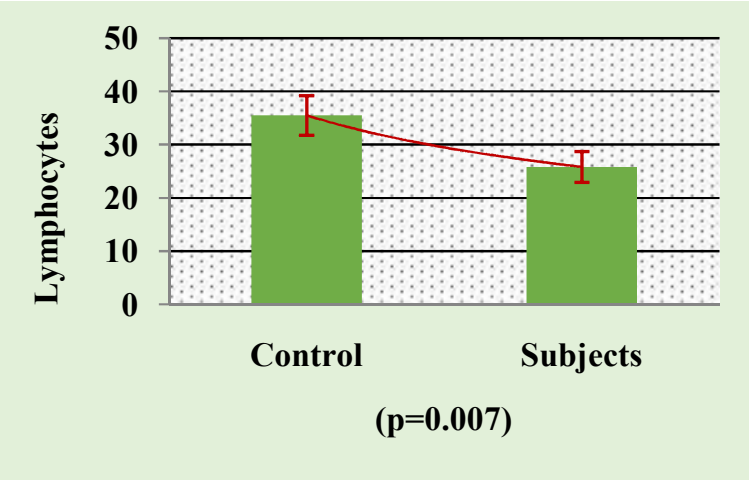

B 


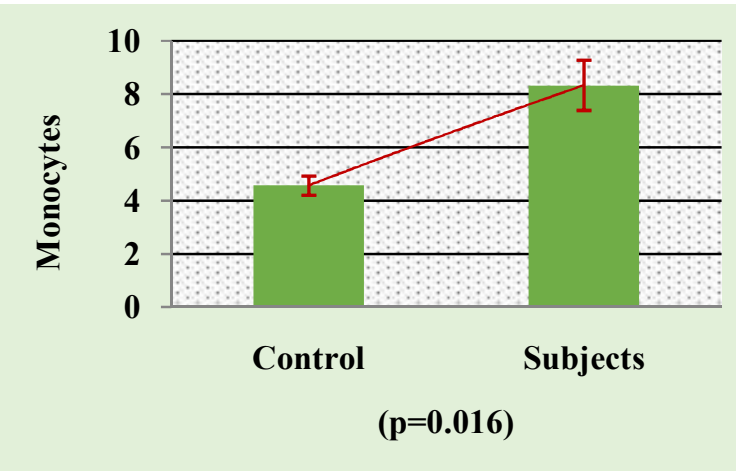

C

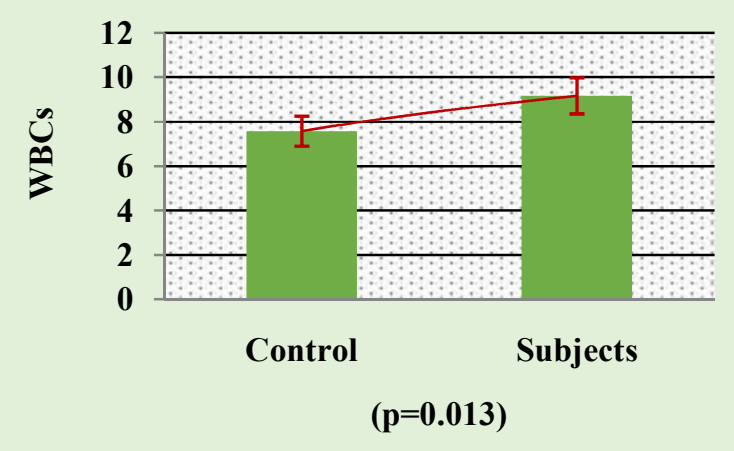

E

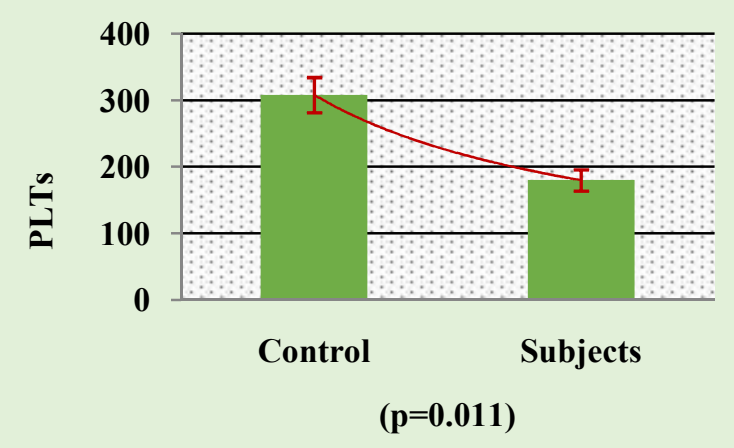

G

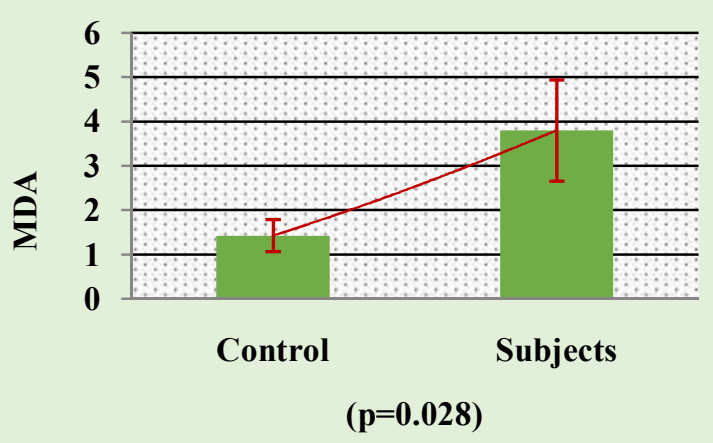

I

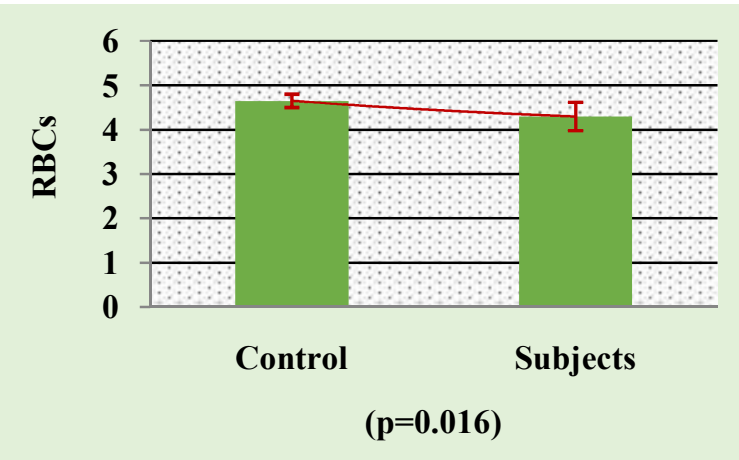

D

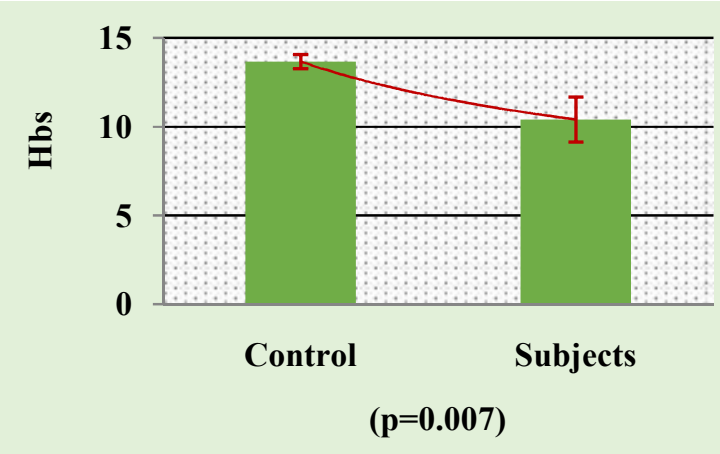

F

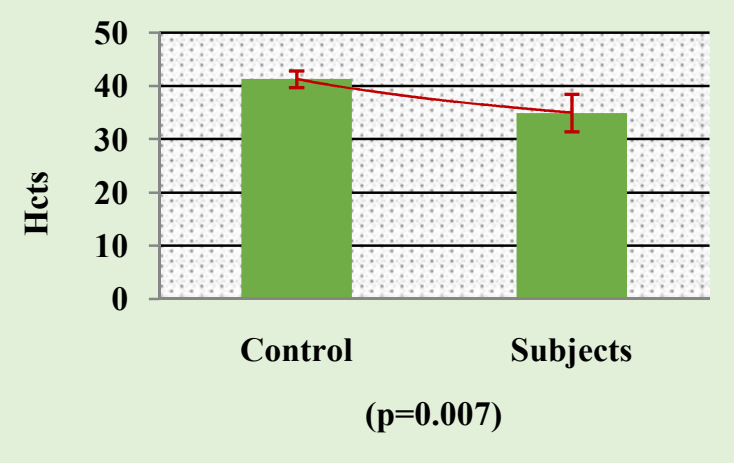

H

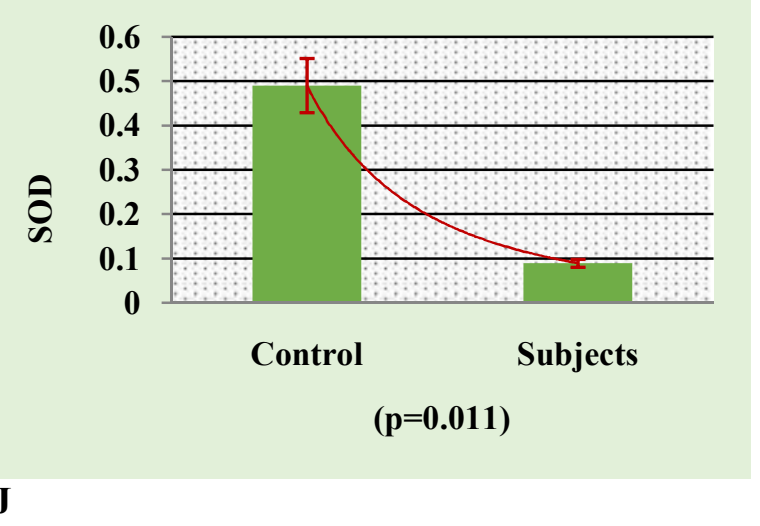




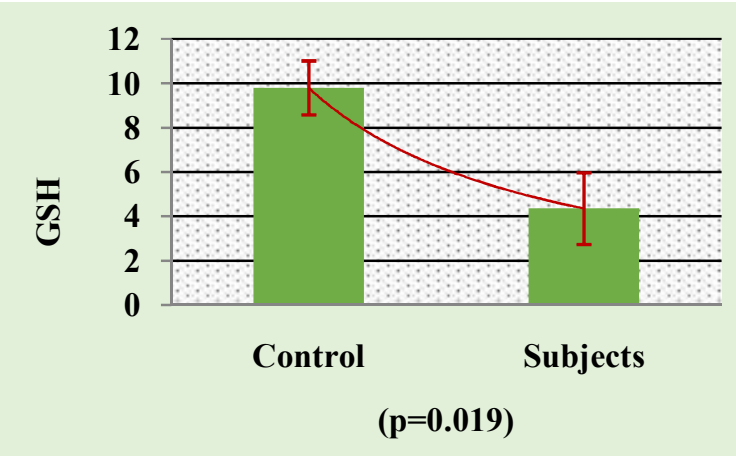

$\mathbf{K}$

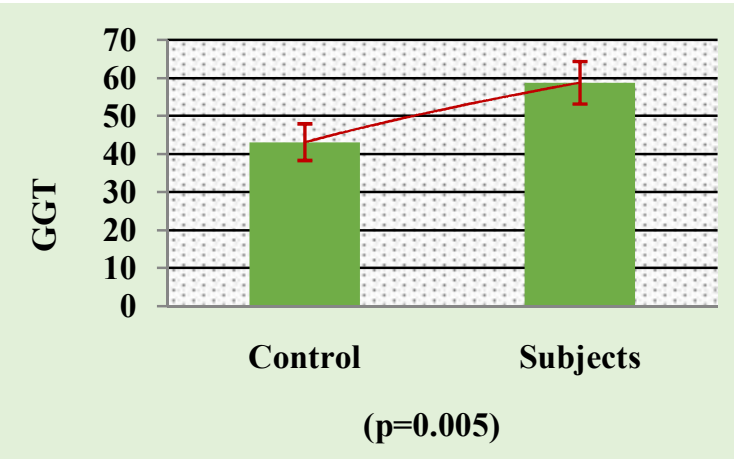

M

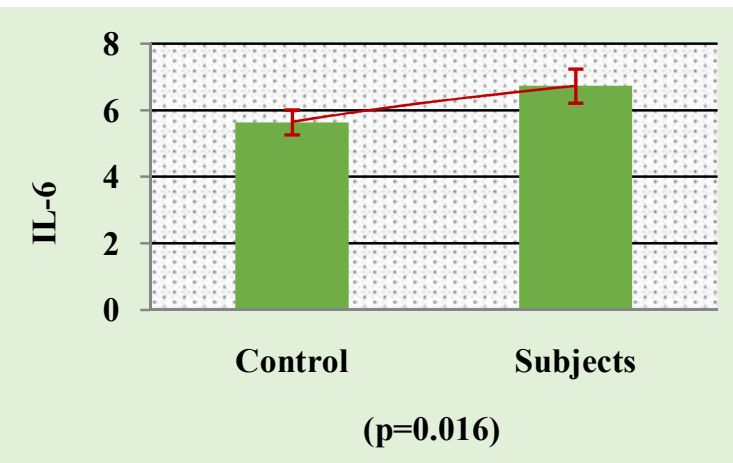

O

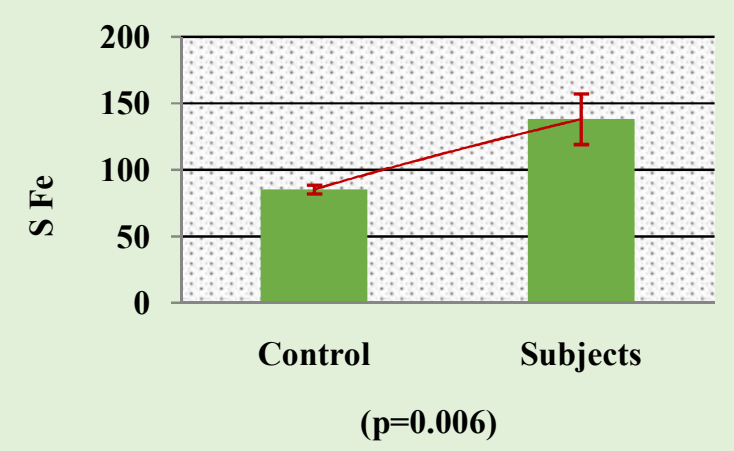

Q

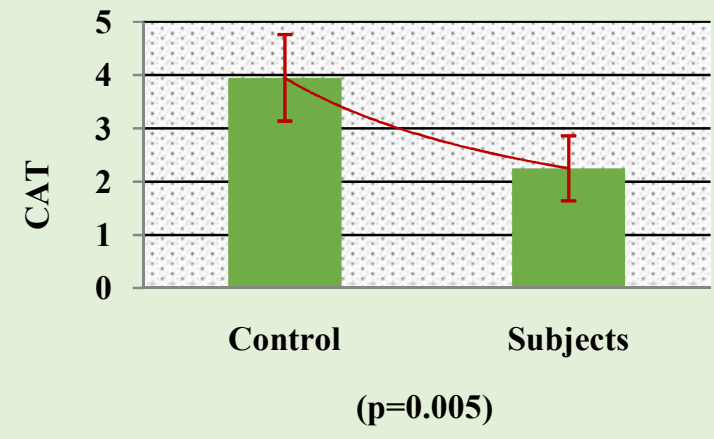

$\mathbf{L}$

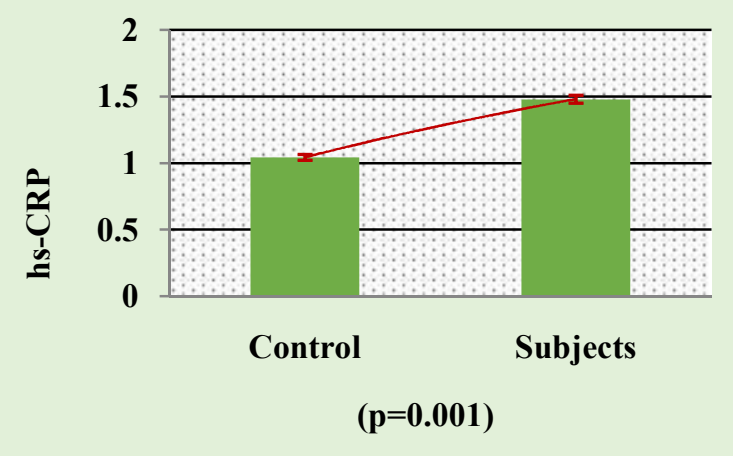

$\mathbf{N}$

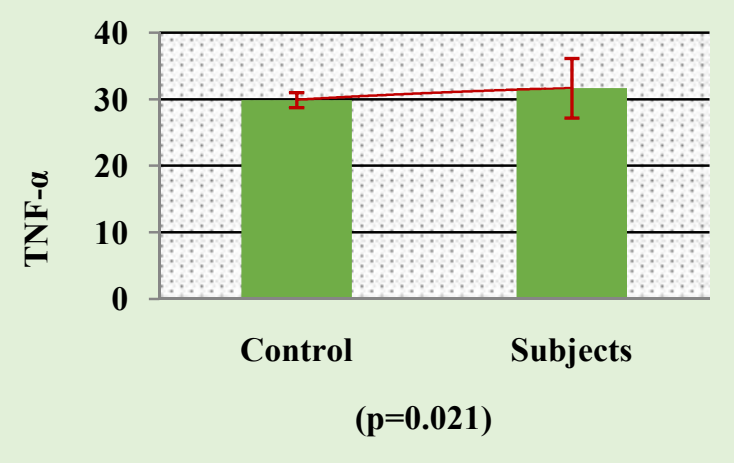

$\mathbf{P}$

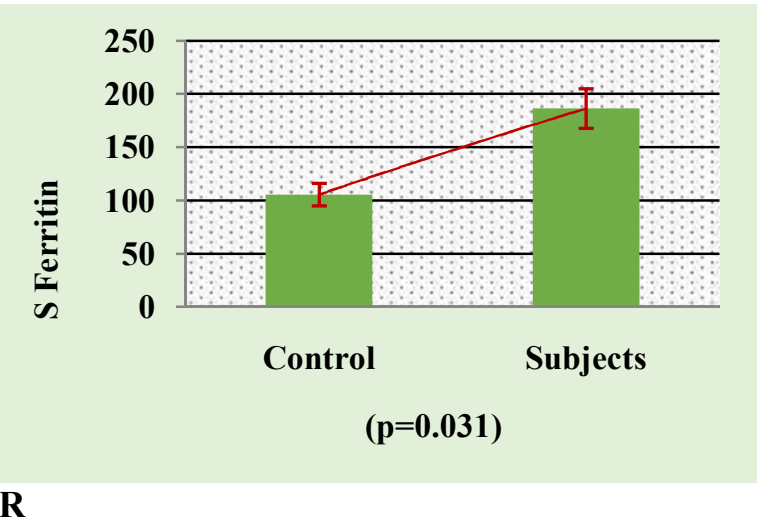




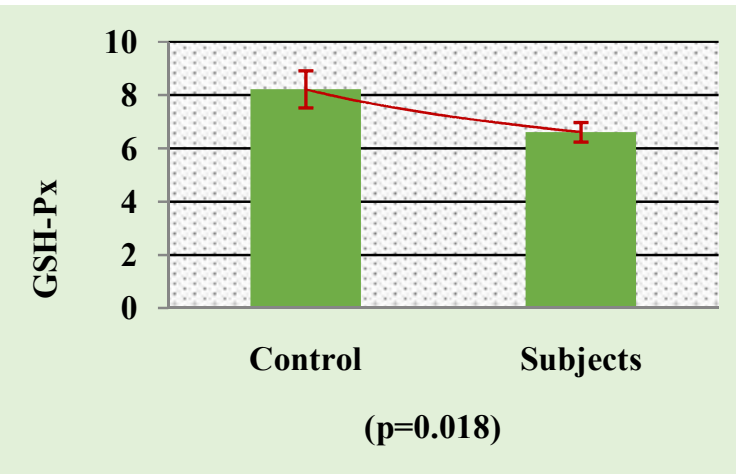

$\mathbf{S}$

\section{DISCUSSION}

Leukemia, lymphoma and myeloma are types of cancers that can affect bone marrow, blood cells, lymph nodes and other organs of lymphatic system. Leukemia is cancer of marrow and blood, characterized by uncontrolled proliferation of immature hematopoietic white blood cells which leads to anemic and other hematologic malignancies. Different molecular factors are involved to cause leukemia. Like STAT5 known as a transcription factor involved in the normal B-Lymphocytes development and in the initiation of leukemia (leukemogenesis). Evidence shows that suppression of STAT5A expression in case of many cell lines result in the high extemporaneous apoptosis expression and an amplified FAS prompted cell demise. But all research is unable to describe the molecular mechanisms which are involved in causing alteration of pre-B cell survival is unclear.

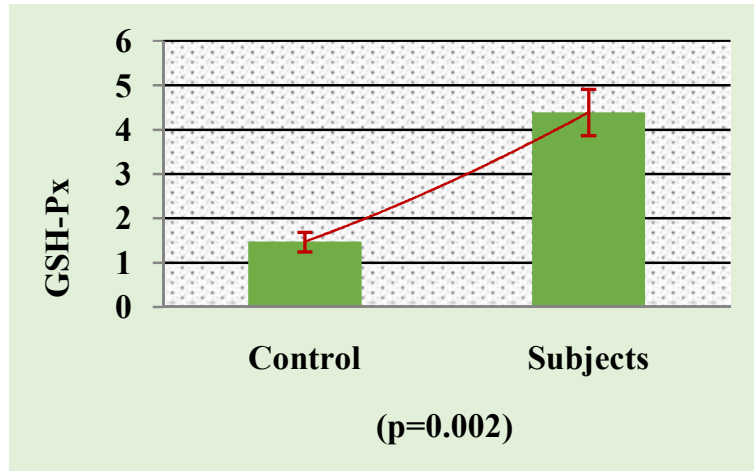

$\mathbf{T}$

Figure 1

There are some facts that the ROS creating oxidative stress and STAT5 are involved in leukemogenesis [23]. Imbalance between production and degradation of reactive oxygen species (ROS) and impaired antioxidation mechanisms lead to the leukemogenesis, which results in the form of myeloid malignancy [24]. Free radicals are responsible for the pathogenesis in a number of diseases. ROS can initiate the lipid peroxidation and other factors which are solely responsible for the pathogenesis not only in leukemias, but also in other cancers [10]. Reactive oxygen species produced by mitochondria or NADPH have been known to affect cell cycle development, movement of cell and signaling of various growth factors in a variety of cells. OS has been proved to be associated with many malignancies and ROS produced in tumor are supposed to enhance cell life, invasion to other organs, tumor progression and 
obstruction to drugs. The relapse and progression of acute myeloid leukemia is linked with increased levels of OS. OS is considered to be a valuable therapeutic objective in leukemic cells but this is still under research that whether OS is supposed to be suppressed or promoted in leukemia. Products of prostaglandins (isoprostanes) are produced by the interaction of free radicals with arachidonic acid and acts as a trustworthy indicator of reactive oxygen damage to lipids. Reactive oxygen species have a significant part in leukemogenesis. Excessive accumulation of ROS leads to hematopoietic malignancies. Hematopoietic malignancy is the basic feature of leukemogenesis in acute and chronic leukemias. Oxidative stress plays a vital protagonist to cause the hematopoietic malignancy, but still it's unclear that whether oxidative stress is involved in the origination, development or repair of the diseases [24]. There are many markers for detection of nuclear and molecular DNA damage but in almost last decade 8-hydroxydeoxyguanosine has become a significant marker for endogenous oxidative stress in pathophysiology, instigation and progression of cancer. This is a product of lipid peroxidation along with MDA (Malondialdehyde). Hence this is mutagenic, causing guanine to thiamine transversion. Excessive production of ROS i.e. oxidative stress is a major progression cause of many hematopoietic malignancies like acute and chronic leukemia, leukemogenesis and which have importance as a therapeutic target [25].

Studies elaborates that the oxidative stress is not only causing drastic effects in case of acute leukemia but also in patients with chronic myeloid leukemia. Low levels of antioxidants and cellular damage in patients with chronic leukemia, supports the idea that oxidative stress has a major part in pathogenesis and prevalence of chronic myeloid leukemia. The expression of BCR$\mathrm{ABL}$, which is due to $\mathrm{Ph}$ chromosome, is responsible for induction of reactive oxygen species in hematopoietic condition. Prominent OS markers i.e. MDA were found in patients of chronic myeloid leukemia. BCR-ABL gene also involved STST5 activation by direct or indirect phosphorylation of JAK2. These signaling pathways transform gene transcription in the nucleus. The phosphorylation of RAS signaling pathway triggers the activation of nuclear factor kappa B, AP-1, c-JUN and cFOS. The activation of these nuclear factors gives rise in MMPs, IL-6, and TNF- $\alpha$ that trigger angiogenesis, apoptosis evasion and tumor metastasis. Stimulation of NFkB 
enhances the levels of i-NOS enzyme which trigger to elevate NO levels and cause angiogenesis. Due to oxidative stress and genetic abnormalities in leukemic patients, there is increased production of reactive oxygen species and inflammatory cytokines (TNF- $\alpha$ and IL-6) which enhance NADPHOxidase as well as activate MAPK signaling pathway. The resultant superoxide $\left(\mathrm{O}_{2}{ }^{-}\right)$ stimulates Monocytes that release inflammatory mediators and also react with NO (Nitric oxide) to synthesize peroxynitrate $\left(\mathrm{ONOO}^{-}\right)$that cause lipid peroxidation in leukemic patients. The superoxide $\left(\mathrm{O}_{2}{ }^{-}\right)$than processed into hydrogen peroxide $\left(\mathrm{H}_{2} \mathrm{O}_{2}\right)$, mediated by Superoxide Oxide (SOD) which is deficient in leukemia. The hydrogen peroxide is converted into hypochlorite $\left(\mathrm{OCl}^{-}\right)$ by MPO (Myeloperoidase) and form oxygen and water molecule with the help of catalase enzyme that is decrease in leukemia. The hypochlorite ion $\left(\mathrm{OCI}^{-}\right)$combines with albumin protein to form AOPPs (Advance glycation end products) which is involved to enhance the activity of NADPH-oxidase. Basically reactive oxygen species (ROS) enhance lipid peroxidation that produce MDA-DNA adducts as well as 8hydroxyguionine (8-oxodG) which is the end product of lipid peroxidation in leukemia. During the second phase of chronic myeloid leukemia OS was relatively high, while the non-enzymatic antioxidants were significantly decreased [26]. The reduced function of GST (Glutathione S transferase) leads to increased risk of conceiving chronic myeloid leukemia and poor diagnosis. Metabolic disturbances that are feature of leukemia elevate reactive oxygen species. Mutations in isocitrate dehydrogenase enzyme increase intracellular OS because mutant enzyme favors conversion of isocitrate to 2 hydroxyglutarate instead of alpha KG. This metabolic shift leads to OS. These eminent ROS caused oxidative damage of biomolecules along with the signaling pathways. Damage to biomolecules occurs due to increased cellular $\mathrm{pH}$, lipid peroxidation and DNA damage and formation of adducts. These conditions are very suitable for progression of leukemia. Normally cells counter to this genotoxic trauma by arresting cell cycle in the initial phase and by inducing apoptosis to safeguard genomic solidity [27-30].

\section{CONFLICT OF INTEREST}

Authors declare no conflict of interest

\section{ACKNOWLEDGEMENTS}

Authors are highly thankful for of all the staff and students of lab-313, The University of Lahore-Pakistan for their valuable contributions in the preparation of 
this manuscript. Here authors are also acknowledged the financial support by the Institute of Molecular biology and Biotechnology, The University of LahorePakistan.

\section{CONCLUSION}

Leukemia are neoplastic disorders, there are several cellular and genetic reasons involved in the progression of the disease. Out of which NK cells reactivity and defective mode of action may contribute as the reason for the leukemia in number of patients. Out study concludes and signifies the importance of antioxidant profile in the progression of disease. As in the subjects having the disorder showed significant decrease in their antioxidant levels such as SOD, CAT, GSH and had increased lipid peroxidation and increased levels of MDA and lipid profile.

\section{REFERENCES}

[1] Hasserjian RP (2013). Acute myeloid leukemia: advances in diagnosis and classification. International journal of laboratory hematology. 35:358-366.

[2] Zuo XL, JM Chen, X Zhou, XZ Li and GY Mei (2006). Levels of selenium, zinc, copper, and antioxidant enzyme activity in patients with leukemia. Biological trace element research. 114:41-53.
[3] Charalambous A and P Vasileiou (2012). Risk factors for childhood leukemia: a comprehensive literature review. Health science journal. 6:432-468.

[4] Mendivil-Perez M, C Velez-Pardo and M Jimenez-Del-Rio (2012). TPEN induces apoptosis independently of zinc chelator activity in a model of acute lymphoblastic leukemia and ex vivo acute leukemia cells through oxidative stress and mitochondria caspase-3- and AIF-dependent pathways. Oxidative medicine and cellular longevity. 313275

[5] Choi, WY, BT Choi, WH Lee, and YH Choi (2008). Sulforaphane generates reactive oxygen species leading to mitochondrial perturbation for apoptosis in human leukemia U937 cells. Biomedicine \& pharmacotherapy. 62:637-644.

[6] Yin L, Z Wu, D Avigan, J Rosenblatt, R Stone, S Kharbanda and D Kufe (2011). MUC1-C oncoprotein suppresses reactive oxygen species-induced terminal differentiation of acute myelogenous leukemia cells. Blood. 117:4863-4870.

[7] Yahya RS, MA Sofan, HM Abdelmasseih, N Saudy and MA SharafEldein (2013). Prognostic implication of BAALC gene expression in adult acute 
myeloid leukemia. Clinical laboratory. 59:621-628.

[8] Shin DY, I Kim, KH Kim, Y Choi and SH Beom(2011). Acute lymphoblastic leukemia in elderly patients: a single institution's experience. The Korean journal of internal medicine. 26:328-339.

[9] Mouna Saadaoui LA, Véronique Salaun, Mohamed Manai and, and S Allouche (2013). Mitochondrial DNA Alterations and Oxidative Stress in Acute Leukemia. The Open Leukemia Journal. 5:1-6

[10] Ahmad R, R Singh, AK Tripathi,, and RK Singh (2012). Leukocyte superoxide dismutase activity in patients with chronic myeloid leukemia. Revista brasileira de hematologia e hemoterapia. 34:394-395.

[11] Kryston TB, AB Georgiev, P Pissis and AG Georgakilas (2011). Role of oxidative stress and DNA damage in human carcinogenesis. Mutation research. 711:193-201.

[12] Xiao Y, P Zou P, J Wang, H Song, J Zou and L Liu (2012). Lower phosphorylation of p38 MAPK blocks the oxidative stress-induced senescence in myeloid leukemic CD34 (+) CD38 (-) cells. Journal of Huazhong University of Science and Technology Medical sciences. 32: 328-333.
[13] Xu J, Z Li, P Xu and Z Yang (2012). Protective effects of leukemia inhibitory factor against oxidative stress during high glucose-induced apoptosis in podocytes. Cell stress \& chaperones. 17:485-493.

[14] Gonzales R, C Auclair, E Voisin, H Gautero, D Dhermy and P Boivin (1984). Superoxide dismutase, catalase, and glutathione peroxidase in red blood cells from patients with malignant diseases. Cancer research. 44:41374139.

[15] Tabe Y, YX Shi, Z Zeng, L Jin and M Shikami,,(2013). TGF-beta-Neutralizing Antibody 1D11 Enhances CytarabineInduced Apoptosis in AML Cells in the Bone Marrow Microenvironment. Plos one. 8:62785.

[16] Kakkar R, J Kalra, SV Mantha and K Prasad (1995). Lipid peroxidation and activity of antioxidant enzymes in diabetic rats. Molecular and cellular biochemistry, 151: 113-119.

[17]Richard MJ, B Portal, J Meo, C Coudray, A Hadjian and A Favier (1992). Malondialdehyde kit evaluated for determining plasma and lipoprotein fractions that react with thiobarbituric acid. Clinical chemistry, 38: 704-709. 
[18] Sarmento-Ribeiro AB, MT Proenca, I Sousa, A Pereira, F Guedes, A Teixeira and CR Oliveira (2012). A possible role for oxidation stress in lymphoid leukaemias and therapeutic failure. Leukemia research. 36:1041-1048.

[19] Devi GS, MH Prasad, I Saraswathi, D Raghu, DN Rao and PP Reddy (2000). Free radicals antioxidant enzymes and lipid peroxidation in different types of leukemias. Clinica chimica acta; international journal of clinical chemistry. 293:53-62.

[20] Santos Sda S, LR Melo, RJ Koifman and S Koifman (2013). Cancer incidence, hospital morbidity, and mortality in young adults in Brazil. Cadernos de saude publica. 29:1029-1040.

[21]Hasserjian RP (2013). Acute myeloid leukemia: advances in diagnosis and classification. International journal of laboratory hematology. 35:358-366.

[22] Fimognari C, M Nusse, R Cesari, R Iori, G Cantelli-Forti and Hrelia Patrizia (2002). Growth inhibition, cell cycle arrest and apoptosis in human T-cell leukemia by isothiocynate Sulforaphane. Carcinogenesis. 23:581-586.

[23] Cholez E, V Debuysscher, J Bourgeais, C Boudot, J Leprince, $\mathrm{F}$ Tron, $\mathrm{B}$ Brassart, A Regnier, E Bissac, E Pecnard
(2012). Evidence for a protective role of the STAT5 transcription factor against oxidative stress in human leukemic preB cells. Leukemia. 26:2390-2397.

[24] Hole PS, RL Darley, and A Tonks (2011). Do reactive oxygen species play a role in myeloid leukemias? Blood. 117:5816-5826.

[25] Jensen CD, G Block, P Buffler, X Ma, S Selvin and S Month (2004). Maternal dietary risk factors in childhood acute lymphoblastic leukemia (United States). Cancer causes \& control CCC. 15:559570.

[26] Imbesi S, C Musolino, A Allegra, A Saija, F Morabito, G Calapai and S Gangemi (2013). Oxidative stress in oncohematologic diseases: an update. Expert review of hematology. 6:317325.

[27]Hockenberry MJ, OA Taylor, PM Gundy, AM Ross, A Pasvogel, D Montgomery, P Ribbeck, K McCarthy and I Moore (2014), F2-Isoprostanes: a measure of oxidative stress in children receiving treatment for leukemia. Biol Res Nurs. 16:303-309.

[28]Zulfiqar HF, Javed A, Afroze B, Ali Q, Akbar K, Nadeem T, Rana MA, Nazar ZA, Nasir IA, Husnain T (2017). HIV diagnosis and treatment through 
advanced technologies. Frontiers in

Public Health. 7; 5:32.

[29] Nadeem T, Khan MA, Ijaz B, Ahmed N, ur Rahman Z, Latif MS, Ali Q, Rana MA (2018). Glycosylation of recombinant anticancer therapeutics in different expression systems with emerging technologies. Cancer Research. 1; 78(11):2787-98.

[30] Imran A, Qamar HY, Ali Q, Naeem H, Mariam RI, Saima AM, Kanwal N, Fawad AL, Sabar MF, Nasir IA (2017). Role of molecular biology in cancer treatment: A review article. Iranian Journal of Public Health. 46(11): 1475. 